\title{
Linx
}

Revue des linguistes de l'université Paris X Nanterre

Approches sociolinguistiques du plan phonique

\section{Variation phonétique et compétences alphabétiques : le traitement des voyelles moyennes antérieures dans la production écrite d'enfants de CE1 et CE2}

\section{Isabelle Malderez}

\section{OpenEdition}

\section{Journals}

Édition électronique

URL : http://journals.openedition.org/linx/826

DOI : $10.4000 / \operatorname{lin} x .826$

ISSN : 2118-9692

Éditeur

Presses universitaires de Paris Nanterre

Édition imprimée

Date de publication : 1 juin 2000

Pagination : 101-117

ISSN : 0246-8743

Référence électronique

Isabelle Malderez, «Variation phonétique et compétences alphabétiques : le traitement des voyelles moyennes antérieures dans la production écrite d'enfants de CE1 et CE2 », Linx [En ligne], 42 | 2000, mis en ligne le 22 juin 2012, consulté le 20 avril 2019. URL : http://journals.openedition.org/linx/826 DOI : $10.4000 / \operatorname{linx} .826$

Ce document a été généré automatiquement le 20 avril 2019.

Département de Sciences du langage, Université Paris Ouest 


\section{Variation phonétique et compétences alphabétiques : le traitement des voyelles moyennes antérieures dans la production écrite d'enfants de CE1 et CE2}

Isabelle Malderez

\section{Production écrite et variation phonétique}

\subsection{Objectifs}

1 Le travail présenté ici s'intègre dans le cadre d'une vaste étude menée sur la production écrite des élèves de l'école primaire en relation avec la variation phonétique. Les objectifs en sont les suivants : $a$ - constituer un corpus de productions écrites d'enfants ; $b$ - étudier le rapport oral/écrit, dans ses composantes parole/écriture, en relation avec l'évolution de la prononciation de la langue: examiner, d'une part, l'impact des variables phonétiques déjà recensées sur la graphie et évaluer, d'autre part, l'impact de nouvelles variables phonétiques sur l'apprentissage de l'orthographe; c- modéliser les relations étudiées en vue de développer un matériel didactique permettant de sensibiliser les jeunes apprenants au « jeu » existant entre prononciation et graphie.

\section{2. État de la question}

2 Pour parler brièvement, la langue française possède deux codes : un code oral et un code écrit. Le premier se transforme de génération en génération, le second est fixé par une académie ou un ministère. Le « désaccord » entre ces deux codes provoque des problèmes 
pédagogiques certains. Il suffit de consulter la table des matières de manuels scolaires tels que le Bled pour établir la liste des erreurs attendues en français de France : doublement ou non des consonnes, graphie des voyelles nasales, confusion des accents, omission ou présence d'un $\underline{\mathrm{e}}$, etc. Ces « fautes " sont liées à des changements phonétiques, en cours de réalisation ou achevés, relevés et étudiés depuis longtemps. Cependant, les enseignants sont souvent confrontés à des fautes d'orthographe atypiques c'est-à-dire des fautes qui ne sont pas prévues dans les exercices de régulation: si "des chevals» est une faute typique (pluriel irrégulier), « des chovaux » ne l'est pas ${ }^{1}$. C'est le cas aussi des troncations graphiques issues de la chute de /R/ ou de /l/ dans les groupes consonantiques finals : "quate cartabes ». La variation phonétique (confusion de sons) entraine donc des fautes lexicales ou grammaticales (confusion de lettres) chez les enfants mais aussi chez les adultes, dans des mots à l'orthographe peu fixée comme les noms de produits : un sujet sur deux écrit « roblochon » pour reblochon (Malderez 1995).

3 Le corpus en cours d'analyse comprend donc, d'une part, l'ensemble de la production en " expression écrite » de 11 enfants scolarisés au CE2 en 1997-98, celle, d'autre part, de 4 enfants scolarisés au CE1 puis au CE2 en 1997-99 et enfin, celle de 2 enfants ayant effectué les deux années au CE1. Chacun des 17 élèves a produit entre 61 et 350 lignes de texte ${ }^{2}$. La classe à deux niveaux se situe dans une école rurale du sud-est de l'Allier. Les pères appartiennent majoritairement aux C.S.P. « ouvriers/employés » (8) et « artisans » (5).

\section{L'enquête sur la scription des voyelles moyennes}

\subsection{Les variables}

4 Certaines oppositions phonologiques, telle la paire /e/-/E/, provoquent des difficultés orthographiques qui perdurent. La grande majorité des élèves échoue par exemple dans le traitement des accents et des finales verbales, et ce, bien après l'école primaire. Les résultats présentés ci-dessous concernent donc l'analyse des essais de scription de cette paire, tout d'abord globalement et ensuite en finales verbales.

\subsubsection{La paire /e/-/ع/ du point de vue phonologique}

5 L'opposition a été longuement étudiée en français contemporain. En syllabe non finale, la tendance est à une distribution complémentaire des variantes: $[\varepsilon]$ apparaît en syllabe entravée et [e] en syllabe ouverte. En ce qui concerne les finales absolues, les enquêtes des fonctionnalistes font apparaître un net maintien de l'opposition, dans ce contexte, chez les adultes (Martinet 1945; Deyhime 1967; Walter 1976) et chez les adolescents (Léon 1973) parisiens. Dans l'étude de Léon, selon les graphies, 60 à $100 \%$ des $/ \varepsilon /$ finaux sont réalisés ouverts, la finale nominale -er obtenant le plus bas score.

Dans le cadre d'une recherche consacrée aux variétés régionales des systèmes phonologiques enfantins (Houdebine 1985), les études de Lefebvre, Goudailler et Boucherit dans le Midi et le Nord de la France ont mis en évidence un système vocalique à 3 niveaux d'aperture chez les élèves entrant au $\mathrm{CP}$. Moins de $10 \%$ des enfants possèdent l'opposition /e/-/ $/ \varepsilon /$ en finale absolue. La voyelle $/ \varepsilon /$ est réalisée fermée ou, au mieux, par un timbre [E]. Par contre, à Paris, Peretz-Juillard a observé que 39 à $67 \%$ des $/ \varepsilon /$ finaux sont réalisés ouverts ou semi-ouverts dans les deux écoles où elle a enquêté. Enfin, Konopczynski a montré que dans l'Est, en particulier à Besançon, les enfants acquièrent 
un système où perdure une opposition de longueur pour les voyelles moyennes fermées / e/ - /e :/, les voyelles moyennes ouvertes étant toujours brèves. Les $/ \varepsilon /$ finaux sont réalisés ouverts dans $100 \%$ des cas à Besançon, et entre $55 \%$ (finale graphique -et) et $80 \%$ (finale graphique -ai) à Nancy. Ainsi, les variations régionales sont importantes et l'influence des finales graphiques sur la prononciation est réelle 3 .

7 Cependant, aucune étude ne décrit le système phonologique des enfants de l'Allier. Ce département, qui recoupe pratiquement les frontières du Bourbonnais, a été rattaché à la région Auvergne à la Révolution française mais fait partie de la zone d'oill. La description linguistique du département figure dans l'atlas régional du Centre. Du point de vue dialectal, le lieu d'enquête, la Montagne Bourbonnaise située au sud-est du département, constitue un carrefour linguistique remarquable entre les domaines occitan (Forez), d'oil (Bourbonnais) et francoprovençal (Roannais) (Escoffier 1958 ; Tuaillon 1976).

\subsubsection{La transcription graphique de /e/}

8 Catach (1995) a montré qu'en français $78 \%$ des /e/ sont transcrits par le graphème $(e)$ et $21 \%$ par (é). Le corpus des productions enfantines permet de dégager 756 mots-références (norme orthographique) pour la paire /e/-/E/ ${ }^{4}$. L'analyse de ces références fait ressortir la répartition suivante pour/e/: $59 \%$ des occurrences sont transcrites par (e), $35 \%$ par (é) et $6 \%$ par (ai). Certains mots anglais du corpus présentent un (e) dans le contexte dévolu à (é) soit le type grapho-phonologique $(E \$)^{5}$. Mais globalement, en français, $(e)$ ne peut transcrire /e/ que dans une syllabe graphique fermée, c'est-à-dire devant les consonnes finales muettes - (EC\#) pied, nez, et, aimer, les, clef; $(E C H \#)$ pieds, clefs - et certains groupes consonantiques internes - (E\$KK) dessert, tennis, essaie ; (Es\$K) descente, Desjardins (tabl. 1).

Tableau 1 : Graphèmes rencontrés dans les mots-références du corpus de productions enfantines selon les types grapho-phonologiques dégagés

\begin{tabular}{|l|l|l|l|l|l|l|l|l|l|l|l|}
\hline Types & \multicolumn{7}{|l|}{ Graphèmes et sous-graphèmes } \\
\hline graph. & e & é & ai & è & ê & ei & ë & aî & a & ay & ey \\
\hline & 2793 & 1156 & 934 & 392 & 102 & 5 & 14 & 30 & 3 & 2 & 5 \\
\hline E\# & & + & + & & & & & & & + & + \\
\hline EC\# & + & + & + & + & + & & & & & & \\
\hline ECH\# & + & & & & & & & & & & \\
\hline Ee\# & & + & & & & & & & & & \\
\hline Ees\# & & + & & & & & & & & & \\
\hline ECK\# & + & & & & & & & & & & \\
\hline EK\# & + & & + & & & & + & & & & \\
\hline EKe\# & & & + & + & + & + & & & + & & \\
\hline
\end{tabular}




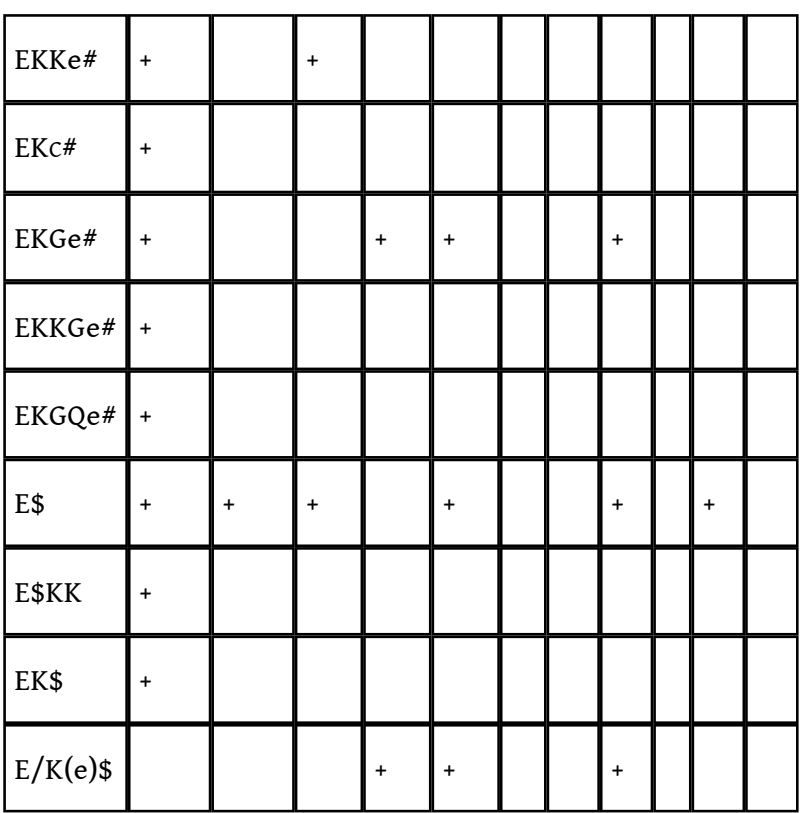

\subsubsection{La transcription graphique de /ع/}

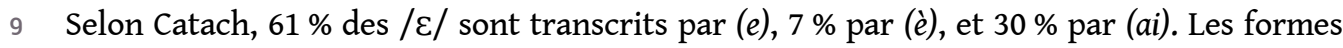
$(\hat{e}),(e i)$ et (ë) sont des graphèmes rares tandis que (â̂) est considéré comme un sousgraphème. Le corpus de productions enfantines comprend quelques mots anglais qui nous obligent à prendre en considération les variantes graphiques (ay), (ey) et (a) - Disney, playmobile, cake. Pour ce phonème, (e) domine avec $44 \%$, suivi de (ai) avec $32 \%$, (è) avec $17 \%$, ( $\hat{e})$ avec $4 \%$ et (ầ) avec $1 \%$. Les variantes (ë), (ei), (a), (ay) et (ey) se partagent les $3 \%$ restants. Malgré sa fréquence, $(e)$ ne transcrit $/ \varepsilon /$ que dans des contextes graphophonologiques particuliers tels que certaines finales muettes - (EC\#) jouet; (ECH\#) est, aspect - les consonnes finales de mot ou de syllabe prononcées - (EK\$) perdu ; (EK\#) mer, sel; (EKGe\#) ferme, herbe; (EKGQe\#) cercle; (Ex\$) Alexandre -, elles-mêmes parfois précédées ou suivies d'une consonne muette - (EKC\#) vert ; (ECK\#) sept -, et enfin les consonnes doubles (EKKe\#) belle, terre; (EKKGe\#) lettre; (E\$KK) erreur.

10 Ainsi le contexte droit est déterminant pour une grande partie des occurrences de la paire / $e /-/ \varepsilon /$ : une erreur sur les consonnes subséquentes ou l'omission d'un accent dans un contexte ne permettant pas la contamination de $(e)$ suffit à faire échouer la transcription graphique des phonèmes.

\subsection{Modalités d'analyse}

\subsubsection{Occurrences analysées ou écartées}

11 Certains essais de scription ont été exclus de l'analyse. Il s'agit, d'une part, de tous les noms de famille et des noms de lieux, les premiers afin de préserver l'anonymat des scripteurs et les seconds parce qu'ils présentent souvent des variantes orthographiques. De même, les prénoms commençant par /e/, orthographié avec (é) en majuscule, ont été exclus à cause de l'impossibilité de contrôler le maintien de l'accent. D'autre part, les essais de scription des jeunes enfants ne sont pas toujours interprétables. Certaines productions ont été écartées car elles n'offrent pas la possibilité de retrouver une 
réalisation du phonème de référence, $\mathrm{y}$ compris son omission: si tous les éléments graphiques de forêt sont présents dans l'essai «frôte ", il est difficile de mettre en relation $/ \varepsilon /$ et la suite « ôte » qui suit le (r). Par contre, des essais tels que

\begin{tabular}{|l|l|}
\hline$(1)$ & "ptale » \\
\hline$(2)$ & "pèta » \\
\hline
\end{tabular}

pour pétales ont été pris en compte : en (1), la lettre (p) [pe] transcrit aussi le phonème /e/ et en (2), la troncation de la deuxième syllabe n'empêche pas l'analyse de la première. Enfin, les alternances d'auxiliaires, /a/ avoir pour / $\varepsilon /$ être, ont été écartées.

\subsubsection{Variables dépendantes}

Cette étude vise à rendre compte, d'une part, de l'adéquation ou non de la transcription graphique d'un phonème donné dans un item donné avec la norme orthographique, et d'autre part, en cas d'erreur orthographique, des différents choix de scription réalisés et de leur aptitude à transcrire le phonème de référence. Deux variables dépendantes ont donc été prises en compte: d'une part la graphie produite et d'autre part le phonème correspondant à la production. Ces variables comportent chacune deux variantes, codées 0 en cas d'adéquation à la référence et 1 en cas contraire, ceci permettant de calculer les taux d'erreurs graphiques ( $\mathrm{Gr}$ ) et de transcription phonémique $(\mathrm{Ph})$.

Du point de vue de la graphie, l'adéquation à la norme a été contrôlée sur le graphème $(E)$ et sur le contexte droit. Ainsi, généralement, il n'a pas été tenu compte des fautes à gauche des graphèmes $(E)$ dans les productions telles que «s'ette" pour cette. Mais certaines segmentations comme «m'et» pour met altèrent $/ \varepsilon /$ et ont donc été comptabilisées.

Du point de vue de la transcription du phonème, les productions aboutissant à une autre voyelle que celle de la référence ont été codées 1 , que le phonème d'arrivée soit l'autre élément de la paire ou encore une autre voyelle. Il faut noter que dans certains cas, il est particulièrement difficile d'établir une correspondance entre la production et un phonème vocalique quelconque.

\subsubsection{Variables indépendantes}

Les variables indépendantes retenues pour l'analyse statistique sont d'une part les variables linguistiques (phonologiques et graphiques) des mots-références et d'autre part les variables sociolinguistiques caractérisant les scripteurs. Le corpus comporte 5436 occurrences d'essais de scription des voyelles moyennes antérieures. La base de référence choisie pour déterminer la catégorie phonémique, la transcription phonétique du Petit Robert (1991), permet de dégager 3155 occurrences de /e/ (58\%) et 2278 de / $\varepsilon /(42 \%)$. Trois occurrences y sont catégorisées par l'archiphonème $/ E /$ et sont donc indéterminées pour la paire $/ \mathrm{e} /-/ \varepsilon /$. La finale phonologique de la syllabe a été prise en compte ainsi que la graphie et le type grapho-phonologique de la référence (voir supra). Parmi les variables caractérisant les scripteurs, ont été retenues le sexe, la classe, l'âge au moment de la production ${ }^{6}$, et enfin le scripteur lui-même. Seules ont été prises en compte dans les analyses statistiques les catégories présentant au moins 10 occurrences. 


\section{Résultats}

\subsection{Résultats globaux}

\subsubsection{Erreurs graphiques $(\mathrm{Gr})$}

Sur les 5436 occurrences, $33 \%$ présentent une erreur graphique sur (E) ou sur le contexte droit (tabl. 2, ${ }^{* *}$ significatif à $98 \%$; ${ }^{*}$ à $95 \%$; ns : non significatif). Les erreurs en finale absolue sont significativement plus nombreuses qu'ailleurs. La classe de /e/ est nettement mieux traitée que celle de $/ \varepsilon /$. Certains types graphiques provoquent plus d'erreurs. Il est évident que la multiplicité des finales graphiques muettes rend la tâche des élèves plus difficile. Les types (Ee\#), (Ees\#), (ECH\#), et (Es\$K) présentent des pourcentages d'erreurs graphiques nettement supérieurs à la moyenne pour /e/, ainsi que $(E C \#)$, (ECH\#), (EKC\#) et (E\$KK) pour / $\varepsilon /$ (tabl. 3a). En ce qui concerne les variables non linguistiques, le facteur classe est opératoire: les CE1 font plus d'erreurs que les CE2. Globalement, filles et garçons réussissent aussi bien dans leurs essais de scription. La variable «âge » n'est pas significative : globalement, les élèves ne font pas de progrès significatifs entre 7 et 10 ans. Enfin, il faut noter que les disparités entre les résultats des différents enfants sont très fortes : le pourcentage d'erreurs graphiques varie de 15 à 50 .

\subsubsection{Erreurs phonémiques $(\mathrm{Ph})$}

Sur les 1807 erreurs graphiques, $76 \%$ présentent aussi une erreur phonémique $(19 \% \mathrm{du}$ total). Les erreurs sont plus fréquentes sur le phonème $/ \varepsilon /$ que sur /e/ et en syllabe ouverte, finale ou non. Parmi les types grapho-phonologiques, (ECH\#), (EC\#) et (E\$KK) provoquent plus d'erreurs que tous les autres pour $/ \varepsilon /$. Pour /e/, seul le type (EC\#) se distingue, et ce, par un taux d'erreurs beaucoup plus faible. En ce qui concerne les scripteurs eux-mêmes, les filles font nettement plus d'erreurs que les garçons et les CE1 plus que les CE2. L'enfant qui réussit le mieux n'en fait que $7 \%$ contre 27 pour ceux qui réussissent le moins bien. Encore une fois, l'âge de l'enfant n'est pas opératoire.

Tableau 2 : Résultats globaux des es-sais de scription de la paire /e/-/ع/

\begin{tabular}{|l|l|l|l|l|l|l|}
\hline catégo. & fact. & N & Gr & p & Ph & p \\
\hline Voy. & e & 3155 & 27 & $* *$ & 11 & $* *$ \\
\hline & E & 2278 & 42 & & 29 & \\
\hline syll. & ou\# & 3702 & 36 & $* *$ & 19 & $* *$ \\
\hline phonol & fer\# & 944 & 27 & & 16 & \\
\hline & ou\$ & 671 & 28 & & 20 & \\
\hline & fer\$ & 113 & 28 & & 12 & \\
\hline sexe & m & 3536 & 33 & $* *$ & 17 & $* *$ \\
\hline
\end{tabular}




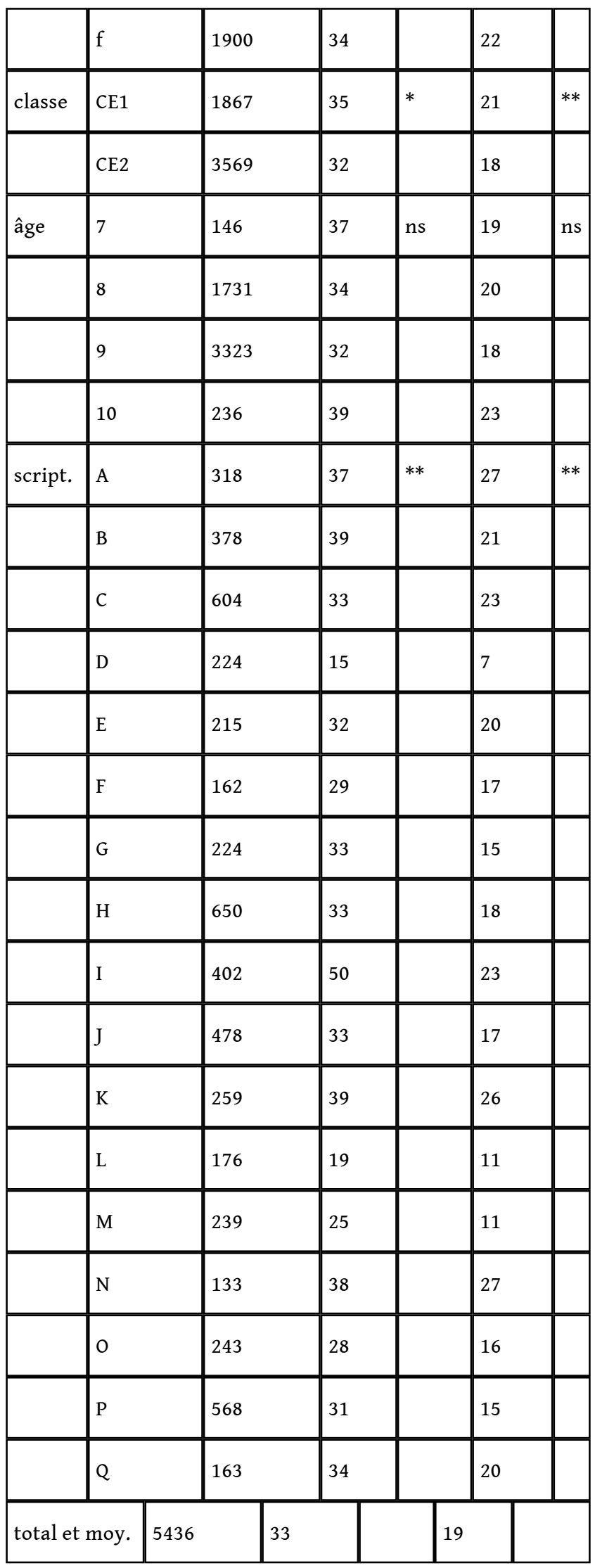


Tableau 3a : Résultats des différents types de référence selon les phonèmes

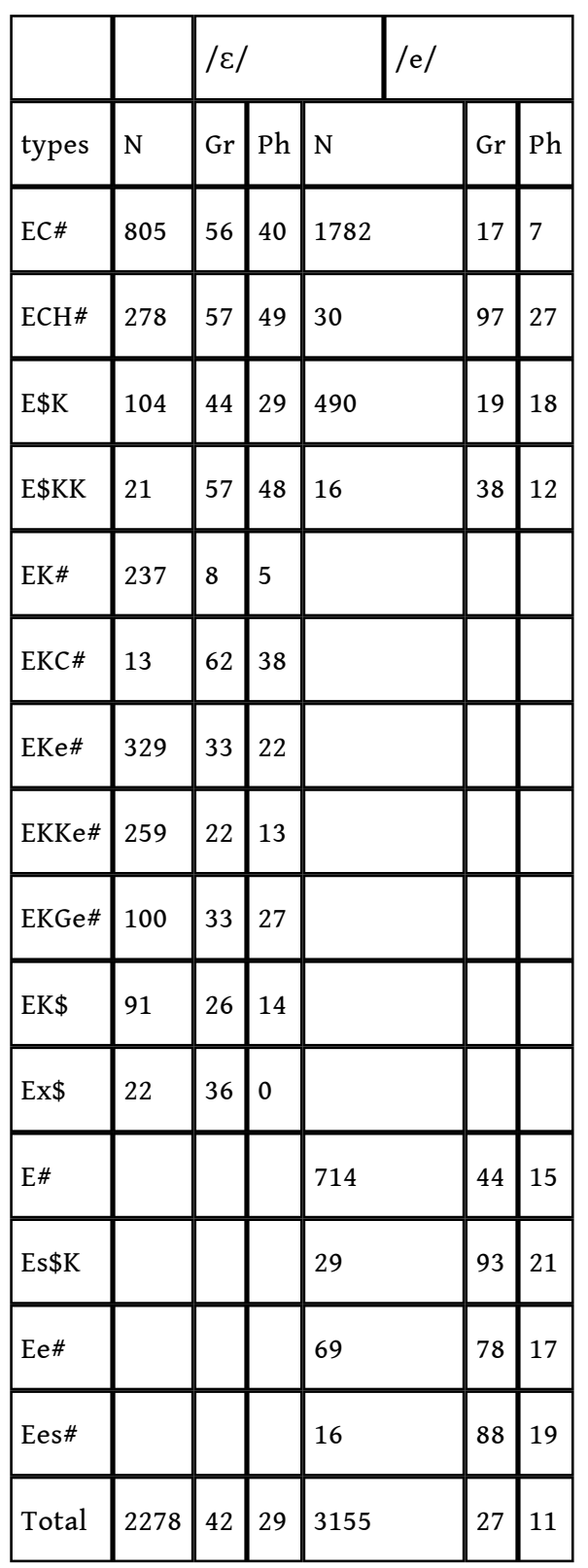

Tableau 3b : Graphèmes de référence selon les phonèmes

\begin{tabular}{|l|l|l|l|l|l|l|l|l|}
\hline & \multicolumn{7}{|c|}{$/ \mathrm{e} /$} & \multicolumn{5}{l|}{$/ \varepsilon /$} \\
\hline gr. & $\mathrm{N}$ & $\mathrm{E}$ & $\mathrm{Ph}$ & $\mathrm{N}$ & $\mathrm{E}$ & $\mathrm{N}$ & $\mathrm{E}$ & $\mathrm{Ph}$ \\
\hline $\mathrm{e}$ & 1796 & 9 & 8 & 997 & 13 & 997 & 13 & 24 \\
\hline é & 1156 & 38 & 18 & & & & & \\
\hline è & & & & 391 & 59 & 507 & 55 & 51 \\
\hline
\end{tabular}




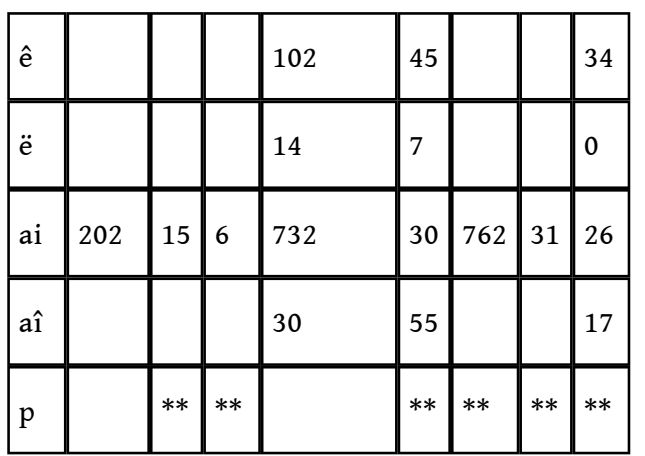

\subsubsection{Erreurs graphiques et phonémiques sur $(E)$}

19 La présence du digraphe (ai) double les taux d'erreurs graphiques sur le seul (E) et celle des graphèmes accentués les quadruple (tabl. $3 b)$. Seul (ë) échappe à ce phénomène. De plus, les erreurs sur (è) sont plus fréquentes que sur (é) ou (ê) $(\mathrm{p}<0,001)$. Dans chacune des deux classes $/ e /$ et $/ \varepsilon /$, les taux d'erreurs phonémiques sur les graphèmes accentués, (é) d'une part, et (è) et (ê) d'autre part, sont supérieurs. Il n'y a pas de différence sur (e) et (ai).

L'analyse des productions erronées met à jour une prédominance des variantes «é » et

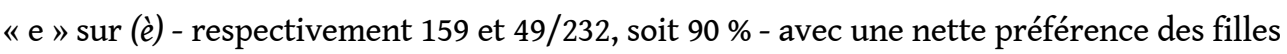
pour «é» (74/81 contre $85 / 127$ pour les garçons). Pour les occurrences phonogrammiques de (é) - participes passés exclus - les variantes «è » et "e » représentent $79 \%(83 / 105)$ des erreurs et « $\mathrm{e}$ » domine largement (72/83). Les choix entre ces deux variantes sont identiques selon les sexes. Parmi les 121 variantes en "e ", certaines correspondent à une simple omission de l'accent et d'autres à des essais de scription avec une consonne contaminante (81 et 41). Elles se répartissent identiquement sur (é) et (è). Les essais avec consonne ne transcrivent pas le phonème de référence dans $35 \%$ des cas pour $/ \varepsilon /$ et $62 \%$ pour /e/, mais la différence n'est pas significative.

\subsection{Les finales verbales}

21 Les finales verbales du français sont le lieu où l'opposition /e/-/E/ est particulièrement productive. C'est aussi celui «qui demande aux élèves le plus lourd travail d'apprentissage et l'effort de contrôle le plus grand » (Brissaud \& Sandon 1999).

Tableau 4 : Catégories et finales graphiques rencontrées en production

\begin{tabular}{|l|l|l|l|}
\hline cat. & N & finales gr. produites & phonèmes \\
\hline é & 406 & é, és, ée, ées,_é, éé, éent & $/ \mathrm{e} /$ \\
\hline er & 329 & er, ér, érr & $/ \mathrm{e} /$ \\
\hline ez & 11 & ez & $/ \mathrm{e} /$ \\
\hline ai & 197 & ai,_ai, /ai, ai\$ & $/ \mathrm{e} / \mathrm{ou} / \varepsilon /$ \\
\hline aiX & 400 & ais,_ais, ait,_ait, aient, aie, aits & $/ \varepsilon /$ \\
\hline
\end{tabular}




\begin{tabular}{|l|l|l|l|}
\hline est & 127 & est, /est & $/ \varepsilon /$ \\
\hline \hline et & 91 & et, ét & $/ \varepsilon /$ \\
\hline e & 67 & e,_e, /e, ee, ent & $/ \partial /$ \\
\hline \hline es & 109 & _es, /es, es\$ & $/ \partial / / \varepsilon / / \mathrm{e} /$ \\
\hline \hline -E & 12 & a, ant, ans, aut, E =0 & $/ \mathrm{a} / / \mathrm{A} / / \mathrm{o} /, \varnothing$ \\
\hline$?$ & 13 & eer, ert, è, èe & divers \\
\hline
\end{tabular}

Le corpus comporte 45 formes différentes pour transcrire les 1762 occurrences de /e/ et / $\varepsilon /$ en finale verbale. Dans un premier temps, afin de simplifier l'analyse, les finales produites ont été regroupées en 11 catégories (tabl. 4). Les formes employées par les enfants sont majoritairement possibles en finale verbale même si elles ne peuvent pas toujours transcrire /e/ ou $/ \varepsilon /$. Quelques rares réalisations relèvent exclusivement $\mathrm{du}$ domaine du nom («aits » faits, « ee » pedigree) ou encore sont étrangères au système du français en finale ("ér ", « érr », « eer ", « è », « èe »).

Globalement, il y a autant d'erreurs graphiques dans la transcription des deux phonèmes mais davantage, si on ne considère que les erreurs sur $(E)$, sur le graphème (é) que sur $(e)$ ou (ai). Aucune des variables non linguistiques, à part le scripteur lui-même, n'est opératoire pour décrire les erreurs graphiques. Par contre, les erreurs phonémiques sont significativement différentes sur les trois graphèmes, et, comme pour l'ensemble du corpus, plus fréquentes chez les filles que chez les garçons et sur / $\varepsilon$ / que sur /e/ (tabl. 5).

\subsubsection{Le traitement des finales en /e/}

Du point de vue graphique, la seule variable non linguistique opératoire est la classe : les CE1 font plus d'erreurs que les CE2 mais elles n'ont pas d'incidence sur le taux de réussite de la transcription phonémique. De ce dernier point de vue, les filles font deux fois plus d'erreurs que les garçons, et les enfants de 7 ans nettement plus que ceux de 8 ou 9 ans (tabl.6). A l'intérieur de la catégorie des finales en /e/, nous pouvons distinguer les participes passés (-É), les infinitifs (-ER), la 1ère personne du futur ou du passé simple et la 1ère personne d'avoir au présent (-AI) et la marque de $5 \mathrm{e}$ personne $(-E Z)^{7}$. Du point de vue graphique, les formes en -AI sont statistiquement mieux traitées que celles en -ER, ellesmêmes mieux traitées que celles en -É. Du point de vue de la correspondance phonémique, les enfants réussissent nettement moins bien sur la classe en -É que sur les deux autres mais il n'y a pas de différence significative entre les résultats des classes -ER et -AI. Ceci nous amène à considérer séparément ces différentes finales.

\section{Le participe passé en -É}

Les finales graphiques muettes des formes en -ée et -és semblent augmenter le taux d'erreurs graphiques (tabl.7). Cependant, si on observe les productions graphiques erronées des enfants, il n'y a pas de différence significative pour les trois finales de référence entre les types produits, en particulier « é » et « er » (tabl. 8 ; t-test apparié, one tail, p: 0,199). En effet, «er» est majoritairement choisi (47\%) et les fautes de genre ou 
nombre ne représentent que $13 \%$ des erreurs. Autrement dit, le taux élevé d'erreurs graphiques n'est pas imputable aux seules finales muettes. D'autre part, le taux d'erreurs phonémiques est sensiblement le même sur les trois formes de participe passé soumises à l'analyse statistique. L'analyse des variables non linguistiques met en évidence un taux d'erreurs graphiques plus élevé chez les filles que chez les garçons sur l'ensemble des finales de référence en $-E ́$, mais identique sur les seules finales en -é (tabl. 8 et 9). Mais dans ces derniers cas d'erreurs, les choix des deux sexes sont différents quant aux graphies produites (tabl. 8 ; Chi2, $p=0,0066$ ). D'autre part, les élèves de CE1 font plus d'erreurs graphiques que les CE2 quelle que soit la finale considérée (-é ou -É).

$\mathrm{Du}$ point de vue phonémique, les filles font, encore une fois, plus d'erreurs que les garçons sur -É et sur le seul -é. Les plus jeunes réussissent moins bien que les plus âgés dans la transcription de /e/ sur l'ensemble de la classe mais pas sur le seul -é. Il faut noter que toutes les occurrences de participes passés chez les jeunes de 7 ans correspondent à des finales en -é.

Tableau 5 : Finales verbales

\begin{tabular}{|l|l|l|l|l|l|l|}
\hline cat. & fact. & N & Gr & p & Ph & p \\
\hline \hline voy. & e & 964 & 52 & ns & 15 & $* *$ \\
\hline & E & 798 & 54 & & 38 & \\
\hline \hline graph & e & 495 & 27 & $* *$ & 35 & $* *$ \\
\hline & é & 559 & 55 & (E) & 19 & \\
\hline & ai & 708 & 27 & & 25 & \\
\hline sexe & m & 1132 & 51 & ns & 22 & $* *$ \\
\hline & f & 630 & 56 & & 31 & \\
\hline \hline classe & ce1 & 642 & 52 & ns & 24 & ns \\
\hline & ce2 & 1120 & 53 & & 26 & \\
\hline âge & 7 & 40 & 60 & ns & 35 & ns \\
\hline \hline & 8 & 547 & 54 & & 25 & \\
\hline & 9 & 1107 & 53 & & 25 & \\
\hline script. & C & 178 & 52 & $* *$ & 28 & $* *$ \\
\hline & I & 122 & 76 & & 31 & \\
\hline & 70 & 33 & & 10 & \\
\hline
\end{tabular}




\begin{tabular}{|c|c|c|c|c|}
\hline & $\mathrm{H}$ & 260 & 50 & 24 \\
\hline & $\mathrm{J}$ & 155 & 50 & 18 \\
\hline & G & 75 & 55 & 16 \\
\hline & 0 & 94 & 43 & 20 \\
\hline & $\mathrm{P}$ & 197 & 55 & 28 \\
\hline & A & 95 & 58 & 40 \\
\hline & B & 111 & 61 & 20 \\
\hline & D & 79 & 25 & 9 \\
\hline & $\mathrm{F}$ & 35 & 54 & 23 \\
\hline & $\mathrm{L}$ & 51 & 35 & 22 \\
\hline & $\mathrm{N}$ & 37 & 70 & 41 \\
\hline & $\mathrm{E}$ & 69 & 65 & 33 \\
\hline & $Q$ & 46 & 72 & 43 \\
\hline & K & 88 & 49 & 31 \\
\hline total et $r$ & moy. & 1762 & 53 & 25 \\
\hline
\end{tabular}

Tableau 6 : Finales en /e/

\begin{tabular}{|l|l|l|l|l|l|l|}
\hline cat. & fact. & $\mathrm{N}$ & $\mathrm{Gr}$ & $\mathrm{p}$ & $\mathrm{Ph}$ & $\mathrm{p}$ \\
\hline gr. & $\mathrm{e}$ & 203 & 43 & $* *$ & 12 & $* *$ \\
\hline & é & 559 & 55 & $(\mathrm{E})$ & 19 & \\
\hline fin. & ER & 199 & 52 & $* *$ & 12 & $* *$ \\
\hline \hline & ai & 202 & 15 & & 5 & \\
\hline E & 559 & 64 & & 19 & \\
\hline sexe & m & 625 & 50 & ns & 11 & $* *$ \\
\hline \hline & AI & 202 & 15 & & 5 & \\
\hline
\end{tabular}




\begin{tabular}{|l|l|l|l|l|l|l|}
\hline cla. & ce1 & 346 & 58 & $* *$ & 17 & ns \\
\hline \hline & ce2 & 618 & 49 & & 13 & \\
\hline âge & 7 & 28 & 61 & ns & 32 & $*$ \\
\hline \hline & 8 & 295 & 53 & & 12 & \\
\hline \hline & 9 & 602 & 51 & & 15 & \\
\hline & 10 & 39 & 46 & & 15 & \\
\hline
\end{tabular}

Tableau 7 : Participes passés en $-\dot{E}$

\begin{tabular}{|l|l|l|l|l|l|l|}
\hline cat & fact. & $\mathrm{N}$ & $\mathrm{Gr}$ & $\mathrm{p}$ & $\mathrm{Ph}$ & $\mathrm{p}$ \\
\hline É & é & 455 & 58 & $* *$ & 19 & $\mathrm{~ns}$ \\
\hline \hline & ée & 41 & 90 & & 27 & \\
\hline sexe & m & 369 & 63 & $*$ & 16 & $*$ \\
\hline \hline és & 56 & 95 & & 11 & \\
\hline classe & ce1 & 195 & 72 & $* *$ & 21 & ns \\
\hline \hline & f 2 & 364 & 60 & & 17 & \\
\hline \hline âge & 7 & 11 & 64 & ns & 45 & ns \\
\hline & 8 & 175 & 67 & & 14 & \\
\hline & 10 & 27 & 44 & & 22 & \\
\hline
\end{tabular}

Tableau 8 : Répartition des choix erronés sur les formes en -É

\begin{tabular}{|l|l|l|l|l|l|l|l|l|l|l|l|}
\hline réf. & production & & & & & & & & & & \\
\hline É & N & é & er & ez & ai & aiX & et & e & es & -E & ? \\
\hline \hline é & 263 & 13 & 133 & 4 & 8 & 45 & 8 & 25 & 18 & 2 & 7 \\
\hline \hline$m$ & 180 & 6 & 99 & 4 & 7 & 29 & 7 & 13 & 13 & 0 & 2 \\
\hline
\end{tabular}




\begin{tabular}{|l|l|l|l|l|l|l|l|l|l|l|l|}
\hline$f$ & 83 & 7 & 34 & 0 & 1 & 16 & 1 & 12 & 5 & 2 & 5 \\
\hline \hline ée & 37 & 11 & 16 & & & 1 & 1 & 7 & & & 1 \\
\hline ées & 7 & 3 & 2 & & & & & 1 & 1 & & \\
\hline \hline és & 53 & 19 & 19 & & & & 3 & 5 & 7 & & \\
\hline T & 360 & 46 & 170 & 4 & 8 & 46 & 12 & 38 & 26 & 2 & 8 \\
\hline$\%$ & 100 & 13 & 47 & 1 & 2 & 13 & 3 & 11 & 7 & 1 & 2 \\
\hline
\end{tabular}

Tableau 9 : Analyse des variables sur les participes passés en -é

\begin{tabular}{|l|l|l|l|l|l|l|}
\hline cat. & fact & $\mathrm{N}$ & $\mathrm{Gr}$ & $\mathrm{p}$ & $\mathrm{Ph}$ & $\mathrm{p}$ \\
\hline sexe & $\mathrm{m}$ & 311 & 58 & $\mathrm{~ns}$ & 16 & $*$ \\
\hline \hline & $\mathrm{f}$ & 144 & 58 & & 24 & \\
\hline cl. & $\mathrm{ce} 1$ & 165 & 68 & $* *$ & 22 & $\mathrm{~ns}$ \\
\hline \hline & $\mathrm{ce} 2$ & 290 & 52 & & 17 & \\
\hline \hline âge & 7 & 11 & 64 & $\mathrm{~ns}$ & 45 & $\mathrm{~ns}$ \\
\hline & 8 & 136 & 60 & & 15 & \\
\hline \hline & 9 & 283 & 58 & & 19 & \\
\hline & 10 & 25 & 40 & & 16 & \\
\hline
\end{tabular}

Tableau 10 : Infinitifs en $-E R$

\begin{tabular}{|l|l|l|l|l|l|l|}
\hline cat. & fact. & $\mathrm{N}$ & $\mathrm{Gr}$ & $\mathrm{p}$ & $\mathrm{Ph}$ & $\mathrm{p}$ \\
\hline sexe & $\mathrm{m}$ & 121 & 42 & $* *$ & 5 & $*$ \\
\hline & $\mathrm{f}$ & 78 & 68 & & 22 & \\
\hline classe & $\mathrm{ce} 1$ & 67 & 57 & $\mathrm{~ns}$ & 12 & $\mathrm{~ns}$ \\
\hline & $\mathrm{ce} 2$ & 132 & 50 & & 11 & \\
\hline âge & 8 & 41 & 56 & $\mathrm{~ns}$ & 15 & $\mathrm{~ns}$ \\
\hline & 9 & 148 & 50 & & 11 & \\
\hline
\end{tabular}


Tableau 11 : Répartition des choix graphiques erronés sur les infinitifs

\begin{tabular}{|l|l|l|l|l|l|l|l|l|l|l|}
\hline & \multicolumn{6}{|c|}{ production } \\
\hline & N & é & er & ez & ai & aix & et & e & es & -E \\
\hline m & 51 & 40 & 1 & 0 & 1 & 0 & 3 & 4 & 2 & 0 \\
\hline f & 53 & 29 & 0 & 2 & 1 & 9 & 2 & 4 & 4 & 2 \\
\hline \hline T & 104 & 69 & 1 & 2 & 2 & 9 & 5 & 8 & 6 & 2 \\
\hline
\end{tabular}

\section{L'infinitif}

Les filles font plus d'erreurs graphiques et phonémiques que les garçons (tabl. 10). Les autres variables ne sont pas opératoires. En cas d'erreur graphique, le choix des enfants se porte très majoritairement sur "é " (tabl.11). Les choix graphiques erronés sont différents selon les sexes. Les finales «aiX», «ez» et " $-\mathrm{E}$ » pour l'infinitif sont exclusivement féminines. Les garçons choisissent « é » plus souvent que les filles.

\section{Le présent du verbe avoir}

Sur les 202 occurrences de la classe -AI, 199 correspondent à la première personne du verbe avoir au présent. Nous ne traiterons donc pas des 3 finales de futur ou de passé simple. La forme j'ai est rapidement acquise par les enfants et elle est la seule de tout le corpus qui ne présente pas de différence significative de traitement graphique entre les différents scripteurs présentant au moins 10 occurrences (tabl. 13). Les élèves de CE1, et en particulier ceux âgés de 7 ans, font nettement plus d'erreurs graphiques que les autres, erreurs qui se traduisent aussi par un taux de réussite moins bon dans la transcription phonémique. Les filles, qui ne réussissent pas plus mal que les garçons en orthographe, échouent plus souvent dans la transcription phonémique. De plus, sur les 29 formes erronées, la répartition des choix graphiques est significativement différente selon les sexes, les filles préférant, dans 7 cas sur 8 , la finale « $\mathrm{e}$ » (tabl. $14 ;$ Chi2 ; $\mathrm{p}=0,0004)$.

Tableau 13 : Résultats pour ai

\begin{tabular}{|l|l|l|l|l|l|l|}
\hline cat. & fact. & $\mathrm{N}$ & $\mathrm{Gr}$ & $\mathrm{p}$ & $\mathrm{Ph}$. & $\mathrm{p}$ \\
\hline sexe & $\mathrm{m}$ & 130 & 16 & $\mathrm{~ns}$ & 3 & $*$ \\
\hline \hline & $\mathrm{f}$ & 69 & 12 & & 10 & \\
\hline classe & $\mathrm{ce} 1$ & 81 & 22 & $* *$ & 10 & $*$ \\
\hline & $\mathrm{ce} 2$ & 118 & 9 & & 3 & \\
\hline âge & 7 & 16 & 56 & $* *$ & 25 & $*$ \\
\hline
\end{tabular}




\begin{tabular}{|l|l|l|l|l|l|l|}
\hline & 8 & 77 & 17 & & 5 & \\
\hline & 9 & 103 & 7 & & 3 & \\
\hline script. & C & 15 & 27 & ns & 27 & $* *$ \\
\hline \hline & I & 11 & 9 & & 0 & \\
\hline \hline & M & 13 & 0 & & 0 & \\
\hline & H & 26 & 15 & & 15 & \\
\hline & J & 30 & 14 & & 0 & \\
\hline & G & 14 & 29 & & 0 & \\
\hline & F & 21 & 29 & & 0 & \\
\hline & 14 & 0 & & 0 & \\
\hline
\end{tabular}

Tableau 14 : Répartition des erreurs graphiques sur ai selon les sexes

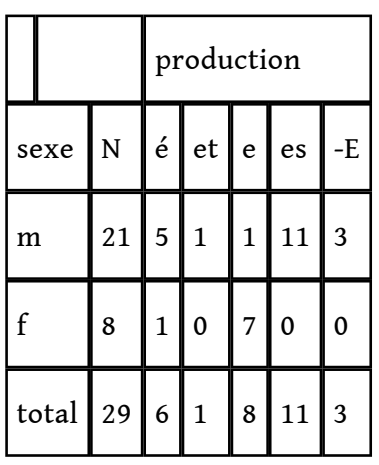

\section{Résumé}

Ce sont les participes passés qui posent le plus de problèmes aux enfants. Quelle que soit la classe des finales verbales en /e/, les filles font plus d'erreurs que les garçons dans la transcription de /e/, ceci pouvant s'expliquer par des choix graphiques erronés toujours différents.

\subsubsection{Le traitement des finales verbales en $/ \varepsilon /$}

Parmi ces finales, on distingue certaines formes d'imparfait et de conditionnel (-AIX), [il] met (-ET), es et est du verbe être au présent $(E S(T))$ et certaines formes de présent, d'impératif ou de participe passé de faire, traire, aller et savoir (-AIC). Les taux d'erreurs graphiques et phonémiques sont statistiquement différents selon les classes dégagées, AIC étant la mieux traitée par les enfants (tabl. 15). Globalement et pour la classe -AIX, les CE2 font plus d'erreurs graphiques et phonémiques que les CE1 mais les différences, graphiques pour est et phonémiques pour -AIC, ne sont pas significatives (tabl. 16). Quelle 
que soit la classe dégagée, la variable "âge » n'est jamais opératoire et le "sexe » seulement pour est (tabl. 17 et 18).

De plus, l'analyse montre une grande disparité des résultats à l'intérieur de chacune des classes en -AIC et en -AIX. Parmi les finales en -AIX, les formes en -ait sont mieux traitées graphiquement que les formes en -ais ou -aient, et les formes en -aient moins bien traitées phonémiquement que chacune des deux autres (tabl. 19). En cas d'erreur, les formes en «é » et «er » dominent (110/189, tabl. 20). Dans la classe -AIC, la différence se situe uniquement dans le domaine graphique, les formes en -ais étant nettement moins bien traitées que les formes en -ait. En cas d'erreur, c'est souvent une forme en $/ \varepsilon /$, et même en « aiX » qui est choisie (52/73). Pour la forme est, les erreurs graphiques relèvent pour une grande part de la confusion homographique avec et (66/154) mais aussi de défauts de segmentation, surtout dans les occurrences de est-ce que écrit « esce que » ou les formes élidées comme n'est écrit « né ».

Tableau 15 : Finales en $/ \varepsilon /$

\begin{tabular}{|l|l|l|l|l|l|l|}
\hline cat. & fact. & $\mathrm{N}$ & Gr. & $\mathrm{p}$ & $\mathrm{Ph}$. & $\mathrm{p}$ \\
\hline grap. & $\mathrm{e}$ & 292 & 15 & $* *$ & 51 & $* *$ \\
\hline \hline & $\mathrm{ai}$ & 506 & 32 & $(\mathrm{E})$ & 33 & \\
\hline fin. & ET & 10 & 90 & $* *$ & 70 & $* *$ \\
\hline & AIX & 289 & 65 & & 51 & \\
\hline \hline & AIC & 217 & 34 & & 9 & \\
\hline & ES(T) & 283 & 57 & & 50 & \\
\hline
\end{tabular}

Tableau 16 : Analyse de la variable « classe » selon les finales

\begin{tabular}{|l|l|l|l|l|l|l|}
\hline & classe & $\mathrm{N}$ & $\mathrm{Gr}$ & $\mathrm{p}$ & $\mathrm{Ph}$ & $\mathrm{p}$ \\
\hline$/ \varepsilon /$ & $\mathrm{ce} 1$ & 296 & 45 & $* *$ & 34 & $*$ \\
\hline & $\mathrm{ce} 2$ & 502 & 60 & & 42 & \\
\hline \hline AIX & $\mathrm{ce} 1$ & 122 & 52 & $* *$ & 43 & $*$ \\
\hline & $\mathrm{ce} 2$ & 167 & 75 & & 56 & \\
\hline AIC & $\mathrm{ce} 1$ & 70 & 24 & $*$ & 10 & $\mathrm{~ns}$ \\
\hline & $\mathrm{ce} 2$ & 147 & 38 & & 9 & \\
\hline est & ce1 & 96 & 49 & $\mathrm{~ns}$ & 42 & $*$ \\
\hline
\end{tabular}




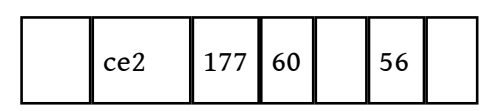

Tableau 17 : Analyse de la variable « sexe » selon les finales

\begin{tabular}{|l|l|l|l|l|l|l|}
\hline finales & sexe & $\mathrm{N}$ & $\mathrm{Gr}$ & $\mathrm{p}$ & $\mathrm{Ph}$ & $\mathrm{p}$ \\
\hline$/ \varepsilon /$ & $\mathrm{m}$ & 507 & 53 & $\mathrm{~ns}$ & 37 & $\mathrm{~ns}$ \\
\hline & $\mathrm{f}$ & 291 & 56 & & 43 & \\
\hline AIX & $\mathrm{m}$ & 180 & 65 & $\mathrm{~ns}$ & 47 & $\mathrm{~ns}$ \\
\hline & $\mathrm{f}$ & 109 & 66 & & 56 & \\
\hline AIC & $\mathrm{m}$ & 137 & 33 & $\mathrm{~ns}$ & 7 & $\mathrm{~ns}$ \\
\hline & $\mathrm{f}$ & 80 & 35 & & 11 & \\
\hline est & $\mathrm{m}$ & 174 & 54 & $*$ & 49 & $\mathrm{~ns}$ \\
\hline & $\mathrm{f}$ & 99 & 61 & & 55 & \\
\hline
\end{tabular}

Tableau 18: Analyse de la variable « âge » selon les finales de référence

\begin{tabular}{|l|l|l|l|l|l|l|}
\hline & âge & $\mathrm{N}$ & $\mathrm{Gr}$ & $\mathrm{p}$ & $\mathrm{Ph}$ & $\mathrm{p}$ \\
\hline total & 7 & 12 & 58 & $\mathrm{~ns}$ & 50 & $\mathrm{~ns}$ \\
\hline$/ \varepsilon /$ & 8 & 252 & 55 & & 40 & \\
\hline & 9 & 505 & 55 & & 39 & \\
\hline & 10 & 29 & 34 & & 31 & \\
\hline AIX & 8 & 101 & 65 & $\mathrm{~ns}$ & 49 & $\mathrm{~ns}$ \\
\hline \hline & 9 & 177 & 65 & & 51 & \\
\hline AIC & 8 & 66 & 33 & $\mathrm{~ns}$ & 11 & $\mathrm{~ns}$ \\
\hline \hline & 9 & 131 & 35 & & 7 & \\
\hline \hline est & 8 & 77 & 57 & $\mathrm{~ns}$ & 55 & $\mathrm{~ns}$ \\
\hline \hline & 9 & 186 & 57 & & 50 & \\
\hline
\end{tabular}




\begin{tabular}{|l|l|l|l|l|l|l|}
\hline & 10 & 10 & 40 & & 40 & \\
\hline
\end{tabular}

Tableau 19: Détail des classes en $-A I X$ et $-A I C$

\begin{tabular}{|l|l|l|l|l|l|l|}
\hline catég. & fact. & $\mathrm{N}$ & $\mathrm{Gr}$ & $\mathrm{p}$ & $\mathrm{Ph}$ & $\mathrm{p}$ \\
\hline AIX & aient & 30 & 97 & $* *$ & 70 & $\mathrm{~ns}$ \\
\hline & ais & 49 & 84 & & 51 & \\
\hline \hline & ait & 210 & 57 & & 48 & \\
\hline AIC & ais & 44 & 66 & $* *$ & 16 & $\mathrm{~ns}$ \\
\hline \hline & ait & 173 & 25 & & 8 & \\
\hline
\end{tabular}

Tableau 20 : Répartition des erreurs graphiques sur les formes en $A I X$ et -AIC

\begin{tabular}{|l|l|l|l|l|l|l|l|l|l|l|l|l|}
\hline & \multicolumn{7}{|c|}{ production } \\
\hline références & N & é & er & ez & ai & aiX & est & et & e & es & -E & $?$ \\
\hline aient & 29 & 13 & 4 & & & 8 & & & 1 & 2 & 1 & \\
\hline ais & 41 & 6 & 8 & & 4 & 15 & & & 1 & 6 & & 1 \\
\hline ait & 119 & 32 & 47 & 5 & 1 & 16 & & 2 & 7 & 3 & 2 & 4 \\
\hline total AIX & 189 & 51 & 59 & 5 & 5 & 39 & 0 & 2 & 9 & 11 & 3 & 5 \\
\hline \hline ait_participe passé & 35 & 5 & 2 & & 5 & 22 & & & & 1 & & \\
\hline ait_présent & 9 & & & & & 8 & 1 & & & & & \\
\hline total ait & 44 & 5 & 2 & & 5 & 30 & 1 & & & 1 & & \\
\hline ais_présent & 8 & 2 & & & & 4 & & & & 1 & 1 & \\
\hline ais_impératif & 21 & & & & & 18 & & 3 & & & \\
\hline total ais & 29 & 2 & & & & 22 & & 3 & & 1 & 1 & \\
\hline total AIC & 73 & 7 & 2 & & 5 & 52 & 1 & 3 & & 2 & 1 & \\
\hline
\end{tabular}




\begin{tabular}{|l|l|l|l|l|l|l|l|l|l|}
\hline & \multicolumn{6}{|c|}{ production } \\
\hline réf. & N & é & ai & aiX & est & et & e & es & -E \\
\hline es & 8 & & & & 6 & 1 & & 1 & \\
\hline \hline est & 154 & 22 & 6 & 7 & 1 & 66 & 4 & 47 & 1 \\
\hline \hline T & 162 & 22 & 6 & 7 & 7 & 67 & 4 & 48 & 1 \\
\hline
\end{tabular}

\section{Résumé}

32 A part pour est, la variable « sexe " n'est jamais opératoire tandis que la " classe » l'est toujours, et ce de manière surprenante : les CE2 réussissent moins bien graphiquement et, à part pour -AIC, dans la transcription du phonème de référence. Étant donné que l'apprentissage systématique de l'imparfait n'intervient qu'au CE2, on peut se demander dans quelle mesure il n'apporte pas plus de confusion dans le choix de scription des finales verbales. D'autre part, la différence de traitement des formes en -AIC et -AIX suggère deux hypothèses: a- les phonogrammes sont plus facilement acquis que les morphogrammes, b- le morphème d'imparfait -ai- est prononcé [e].

\section{Discussion}

\subsection{Orthographe et phonologie}

Les essais de scription des élèves nous permettent de faire quelques hypothèses quant à la nature de l'opposition dans leur système phonologique.

Tout d'abord, aucun mot du corpus ne contient la voyelle /e/ en syllabe fermée. Les erreurs de transcription phonémique sur $/ \varepsilon / \mathrm{y}$ sont peu nombreuses et ne se différencient pas sur_C\$ et _C\# (12 et 16\%). D'autre part, une distribution complémentaire des deux voyelles en syllabe ouverte interne impliquerait l'absence de $[\varepsilon]$. Le corpus contient 126 occurrences de cette voyelle dans les mots-références contre 542 /e/. Les taux d'erreurs dans la transcription phonémique sont supérieurs sur $/ \varepsilon /$ (32\% 18 \%). En cas d'erreur, le phonème d'arrivée est majoritairement l'autre élément de la paire pour $/ \varepsilon /(70 \%)$ et un autre phonème pour /e/ (65\%). Pour / $\varepsilon /$, le graphème erroné d'arrivée est souvent (é) tandis que pour /e/ il s'agit surtout d'une omission de l'accent sur (é) ou d'une consonne double simplifiée. On peut donc penser que, dans le système phonologique de ces enfants, la variante [e] domine dans ce contexte. Enfin, en finale absolue, les pourcentages d'erreurs dans la transcription phonémique de $/ \varepsilon /$ sont beaucoup plus élevés, que l'on considère l'ensemble des occurrences (42 10), les seules finales verbales (38 15) ou les seules finales non verbales (55 7). En cas d'erreur, le phonème d'arrivée est, pour les deux phonèmes, majoritairement l'autre élément de la paire de référence, mais la répartition est différente ( $94 \%$ pour $/ \varepsilon /$ et $64 \%$ pour $/ \mathrm{e} /$ ). Si l'opposition se maintenait bien en finale absolue, les taux de réussite dans la 
transcription phonémique devraient être identiques sur les deux voyelles. Tel n'est pas le cas et les résultats confirment donc les conclusions des études sur les systèmes phonologiques enfantins hors agglomération parisienne.

\subsection{La variable sexe}

Gauchat (1905) a montré que les femmes sont plus sensibles aux modèles de prestige et ont adopté le français au détriment du patois avant les hommes. Labov (1972) a lui mis en évidence que, pour certaines variations, elles sont capables de beaucoup plus de contrôle dans les styles les plus formels tout en employant les formes les plus novatrices en discours familier. Cependant, la différence de comportement langagier entre locuteurs féminins et masculins a peu été étudiée dans le cadre des recherches phonologiques françaises. En ce qui concerne les adultes, Mettas (1973) a mis en évidence des réalisations extrêmes - très ouvertes - de $/ \varepsilon /$ chez les jeunes femmes issues de la bourgeoisie parisienne (15-35 ans). On peut rapprocher ce phénomène du changement de type lermes $>$ larmes dont les femmes furent les initiatrices selon les grammairiens. Mais Mettas ne compare pas les réalisations des femmes et des hommes de ce même sociolecte. Houdebine (1979) a montré qu'en Anjou les femmes maintiennent mieux la distinction / $\varepsilon /-/ e /$ que les hommes dans la mesure où elles produisent plus de réalisations ouvertes de $/ \varepsilon /$ en finale, tout comme les enseignants. Du côté des jeunes locuteurs, Baraduc \& al. (1989) n'ont pas trouvé de différence entre filles et garçons dans les réalisations des deux voyelles antérieures, contrairement à ce qu'ils ont pu mettre en évidence pour les autres oppositions de voyelles moyennes.

Dans le corpus de production écrite, les résultats identiques sur les erreurs graphiques prouvent que les filles de la population étudiée ne sont pas plus «mauvaises" en orthographe que les garçons, ni globalement ni sur les seules finales verbales. Cependant, hormis sur les finales verbales en $/ \varepsilon /$, elles réussissent toujours moins bien dans la transcription phonémique des voyelles de référence $(/ \varepsilon /: 34 \sim 27 \%$ d'erreurs**; /e/: $13 \sim 10 \% *)$. Ceci nous permet d'émettre l'hypothèse que les filles et les garçons de la population étudiée ont un comportement langagier différencié pour l'opposition en termes de réalisations plus fermées pour $/ \varepsilon /$ chez les filles. Les textes du corpus étant des "brouillons", donc peu formels pour des productions scolaires, on peut les rapprocher, sur l'échelle stylistique, de l'oral familier, lieu où les femmes sont parfois plus en avance que les hommes pour certains changements.

\section{BIBLIOGRAPHIE}

BARADUC, J. ; BERGOUNIOUX, G. ; CASTELLOTI, V. ; DUMONT, C. \& LANSARI, M-H. (1989), « Le statut linguistique des voyelles moyennes ", Langage et Société, 49, 5-24.

BRISSAUD, C. \& SANDON, J-M. (1999), « L'acquisition des formes verbales en /E/ à l'école élémentaire et au collège ", Langue française, 124, 40-57. 
CATACH, N. (1995), L'orthographe française, Nathan.

CHEVROT, J-P. \& MALDEREZ, I. (1999), « L'effet Buben : de la linguistique diachronique à l'approche cognitive (et retour) », Langue française, 124, 104-125.

DEYHIME, G. (1967), « Enquête sur la phonologie du français contemporain », La linguistique, I, 97-108, et II, 57-84.

ESCOFFIER, S. (1958), La rencontre de la langue d'oil et de la langue d'oc et du franco-provençal entre Loire et Allier. Limites phonétiques et morphologiques, Belles-Lettres.

GAUCHAT, L. (1905), L'unité phonétique dans le patois d'une commune,Max Niemeyer.

HOUDEBINE, A.-M. (1979), «L'opposition d'aperture /e/-/E/ en français contemporain », La

Linguistique, 15/I, 111-125.

HOUDEBINE, A.-M. (éd.) (1985), La phonologie de l'enfant français de six ans, Buske.

Labov, W. (1972), Sociolinguistics Patterns, Philadelphia. University of Pennsylvania Press (trad. fr. (1976), Sociolinguistique, Editions de Minuit)

LÉON, P. (1973), « Modèle standard et système vocalique du français populaire des jeunes

parisiens ", in Trends in Canadian applied linguistics, G. Rondeau (éd.), C.E.C., 55-79.

MALDEREZ, I. (1995), Contribution à la synchronie dynamique du français contemporain : le cas des voyelles orales arrondies, Thèse de Doctorat, Université Paris 7.

MARTINET, A. (1945), La prononciation du français contemporain, Droz.

METTAS, o. (1973), «Les réalisations vocaliques d'un sociolecte parisien », Travaux de l'Institut de Phonétique de Strasbourg, 5, 1-11.

TUAILLON, G. (1976), Comportements de recherche en dialectologie française., Éd. du CNRS.

WALTER, H. (1976), La dynamique des phonèmes dans le lexique français contemporain, FranceExpansion.

\section{NOTES}

1. Dans l'ensemble de l'article les formes entre guillemets renvoient aux productions rencontrées et les formes en italiques à la norme orthographique.

2. Les textes composant le corpus ont été produits en "premier jet». J'ai longuement insisté auprès des enfants sur le fait que le contenu m'importait plus que la forme, et que la correction n'intervenait qu'après. Je ne leur ai jamais donné l'orthographe des mots sur lesquels ils hésitaient et je pense que bon nombre d'erreurs graphiques n'auraient pas été produites au cours de dictées. Si l'homogénéité stylistique du corpus ne permet pas l'analyse statistique de la variable situationnelle, il fournit un échantillon de la production la moins surveillée qu'il soit possible de récolter dans le cadre de l'école - à part les « petits mots".

3. Pour un état de la question de l'influence de l'orthographe sur la prononciation, voir chevrot \& Malderez (1999).

4. Toutes les formes différentes ont été comptabilisées. On a distingué les formes verbales monosyllabiques dans les contextes d'élision: l'ai et n'ai sont comptées comme deux formes différentes de même que s'est et c'est. Le même choix a été opéré pour les formes identiques appartenant à des catégories grammaticales différentes : été (nom) et été (participe passé). 
5. Dans les types grapho-phonologiques, les lettres $\mathrm{C}$ et $\mathrm{H}$ désignent n'importe quelle consonne muette, $\mathrm{K}, \mathrm{G}$ et $\mathrm{Q}$ les consonnes prononcées et $\mathrm{E}$ l'archigraphème. Les signes \$ et \# renvoient à la structure phonologique de la syllabe.

6. L'appartenance à la classe « 8 ans » signifie que l'enfant avait entre 7,5 ans et 8,4 ans, limites comprises, au moment où il a produit l'occurrence.

7. Nous ne traitons pas ici des formes de référence en -EZ. Pour indication, les quatre occurrences sont mal orthographiées et une d'entre elles présente une erreur phonémique.

\section{RÉSUMÉS}

Cet article présente la méthodologie adoptée pour rendre compte du traitement orthographique de la paire $/ e /-/ \varepsilon /$ dans la production scolaire écrite d'enfants âgés de 7 à 9 ans. Il décrit les erreurs graphiques (avec et sans altération phonémique) globalement puis sur les seules finales verbales. L'analyse statistique montre que les erreurs sur $/ \varepsilon$ / sont les plus fréquentes et d'autre part que les filles, qui font autant d'erreurs graphiques que les garçons, réussissent moins bien dans la transcription des phonèmes. Ceci suggère que, dans le système phonologique enfantin, / $\varepsilon /$ tend vers [e] en finale absolue et en syllabe interne ouverte, et ce surtout chez les filles.

This paper presents the methodology that is adopted to analyze the spelling of $/ e /-/ \varepsilon /$ in school writing of 7-9 years old children. It describes spelling errors (with and without phonological alteration) globally and in the verb finals. Statistical analysis shows that spelling errors on $/ \varepsilon /$ are more frequent than on /e/. Moreover, girls, who do as many spelling errors as boys, fail more in phonemic transcription. This suggests that $/ \varepsilon /$ tends towards [e] in childrens' phonological system in absolute finals and open internal syllabe, especially in girls' production.

\section{AUTEUR}

\section{ISABELLE MALDEREZ}

Paris 7

Ribbes

15170 Celles

malderez@wanadoo.fr 